\title{
Panorama of intimate partner violence against women in Brazil and its association with self- perception of health: findings from a national representative survey
}

Marcos Signorelli ( $\sim$ signorelli.marcos@gmail.com )

Federal University of Paraná

Felipe Granado de Souza

Federal University of São Paulo

Raimundo V. B. Pinheiro Junior

Federal University of São Paulo

Juliana Valente

Federal University of São Paulo

Solange Andreoni

Federal University of São Paulo

Leandro F. M. Rezende

Federal University of São Paulo

Zila Van Der Meer Sanchez

Federal University of São Paulo

\section{Research Article}

Keywords: Intimate Partner Violence, Violence against Women, Brazil, Health Care, Population Studies in Public Health

Posted Date: February 4th, 2022

DOI: https://doi.org/10.21203/rs.3.rs-1262159/v1

License: (c) (i) This work is licensed under a Creative Commons Attribution 4.0 International License. Read Full License 


\section{Abstract}

Objective: This study aimed to describe the prevalence of Intimate Partner Violence (IPV) and its subtypes against adult women in Brazil, as well as their health consequences, and the use of health services resulting from IPV. We also investigated the association of IPV with self-perceived health in victimized women.

Methods: Cross-sectional study including 34,334 women aged 18 to 59 years from the 2019 Brazilian National Health Survey. For the analyses, the prevalence of IPV and its types in the last 12 months, health consequences, and use of health services by these women were calculated. Logistic regression models were performed to estimate the association of sociodemographic characteristics and self-perceived health status with IPV.

Results: The prevalence of IPV against Brazilian adult women in the last twelve months was $7.6 \%$ (95\% Cl: [7.0; 8.2]). Women aged 18 to 39 years, living with a partner, single or divorced, with complete high school or incomplete higher education and income of up to 1 minimum wage, had higher odds of suffer IPV. As health consequences, approximately $69 \%$ of the women who suffered IPV reported psychological consequences. Among those who reported health consequences, $13.9 \%$ sought health care, mainly in primary or secondary health care in the Brazilian Public Unified Health System (SUS) (41.9\%). Regarding the self-perceived health variables, women who reported eating problems $(\mathrm{OR}=1.29 ; 95 \% \mathrm{Cl}[1.01 ; 1.65])$, lack of interest/absence of pleasure (OR=1.41; 95\% Cl $[1.11 ; 1.79])$, depressive feeling ( $\mathrm{OR}=1.39 ; 95 \% \mathrm{Cl}[1.03 ; 1.88])$, feeling of failure $(\mathrm{OR}=1.75 ; 95 \% \mathrm{Cl}[1.36 ; 2.24])$ and suicidal thinking $(\mathrm{OR}=1.73 ; 95 \% \mathrm{Cl}[1.25 ; 2.41])$ had greater odds of reporting IPV, when compared to those who did not report these same perceptions.

Conclusion: The results show that younger, divorced or single, low-income women with eating and mental health problems were more likely to suffer IPV. IPV often led to health problems, and many abused women sought support from public health services due to IPV. Therefore, health professionals must be trained to identify and care for these women, thereby acting as allies in preventing and reducing violence against women in Brazil.

\section{Introduction}

The physical, sexual, and emotional abuse resulting from intimate partner violence (IPV) against women negatively impacts the health of these women and their children ${ }^{1-4}$. The consequences of IPV for women include physical injuries, chronic pain, disfigurement, permanent disabilities, depression, anxiety, posttraumatic stress disorder (PTSD), gastrointestinal and gynecological signs, including sexually transmitted diseases $^{4-6}$. IPV, as well as other forms of violence practiced by familiar people, can also have negative consequences on the perception of the health of victimized women ${ }^{7,8}$, including reports of difficulty for walking, dealing with daily activities, pain, memory loss, and dizziness, among other complaints ${ }^{9}$.

A multicenter study by the World Health Organization (WHO) conducted in ten countries estimated that $15 \%$ (Japan) to $71 \%$ (Ethiopia) of women have suffered physical and/or sexual violence by an intimate partner at some point in their lives, while 4 to $54 \%$, in the same evaluated countries, declared to have suffered it in 
the last twelve months ${ }^{10}$. IPV against women is more prevalent and challenging in low- and middle-income countries (LMIC), with fewer resources available to deal with the problem ${ }^{11,12}$. Brazil stands out negatively in this scenario; the country is among those with the highest figures of femicide in the world ${ }^{13,14}$, a crime usually preceded by repeated episodes of IPV ${ }^{15}$.

Brazil is far from reaching the fifth Sustainable Development Goal (SDG) of the United Nations, which aims at gender equality, empowering women and girls, and eliminating all forms of violence against them ${ }^{16}$. Recent WHO estimates (2018) indicate the prevalence of physical and/or sexual IPV in the last 12 months against Brazilian women aged $15-49$ years, which equals $6 \%(95 \% \mathrm{Cl} 4-10 \%)^{12}$. A study preliminarily analyzed data from the 2019 Brazilian National Health Survey (PNS), which includes a module on violence, revealing that the prevalence of IPV in adult women (18 to 59 years old) was $7.6 \%$, with IPV more associated with younger and low-income women ${ }^{17}$.

However, this study ${ }^{17}$ did not analyze the consequences of IPV for women's health, the search for/use of health services resulting from IPV, nor the association of IPV with self-perceived health. PNS 2019 is the most recent and renowned as the largest and most comprehensive health survey conducted in Brazil, providing information on determinants and health needs of the population, and access to health services, allowing robust analyses to inform health policies and actions ${ }^{17-19}$.

One of the first steps in reducing IPV is knowing how often it occurs and its associated factors and consequences for women's lives. The WHO has recommended nationwide surveys to measure the impacts of violence on women and health systems and services ${ }^{20,21}$.

In this study, we described the prevalence of the types of IPV suffered by adult Brazilian women in the last 12 months, the consequences of IPV on their health, and the search for health care. In addition, we sought to investigate the association between self-perception of women's health status and exposure to IPV to identify predictor variables of exposure to IPV that can help recognize abused women in health services.

\section{Methods}

\section{Type of study, population, and sample}

This is a cross-sectional study using data from the PNS 2019, the largest and most recent epidemiological survey including a representative sample of non-institutionalized people aged 15 or over on health status, lifestyle, access, and use of health services in Brazil ${ }^{19,22}$. PNS 2019 used complex cluster sampling, defined from a Master Sample, in three stages of selection: (1) census tracts or aggregate of sectors - Primary Sampling Units (UPA); (2) households, selected in each UPA; and (3) resident aged 15 or over, selected by simple sampling, in each household. 108,525 households were selected, of which 94,114 individuals agreed to participate (response rate of 93.6\%). For this study, only women, aged between 18 and 59 years old were included, totaling 34,334 respondents. A previous study by Stopa et al. ${ }^{22}$ describes more information about the PNS 2019 sampling plan and data analysis. 


\section{Intimate partner violence, health consequences, and access to health services}

The IPV was defined based on specific questions of Psychological, Verbal, Digital/Technological, Economic, Physical, Sexual or Threat Violence when committed by an "Intimate Partner" (Spouse/partner, Exspouse/ex-partner, boyfriend/girlfriend, and ex-boyfriend/ex-girlfriend). Thus, the occurrence of IPV was informed by answering "Yes" to any questions related to the types of violence practiced and attributed to the intimate partner; answering "No" to all questions informed the absence of IPV.

For analysis of IPV, the following questions were reported: "In the last 12 months, has anyone [...]": "[...] offended, humiliated or ridiculed you in front of other people?" (Psychological violence); "[...] yelled at you or called you names?" (Verbal Violence); "[...] used social media or cell phones to threaten, offend, curse or expose your images without your consent?" (Digital/Technological Violence); “[...] verbally threatened to hurt you or someone important to you?" (Threat); "[...] destroyed something of yours on purpose?" (Economic Violence); "[...] slapped you?", "[...] pushed you, held you tightly or threw something at you intending to hurt?", "[...] punched, kicked or dragged you by the hair?", "[...] tried to or in fact strangled, choked or burned you on purpose?", "[...] threatened or injured you with a knife, firearm or any other weapon or object?" (Physical Violence); “[...] touched, manipulated, kissed or exposed parts of your body against your will?"; "[...] threatened or forced you to have sex or any other sexual acts against your will?" (Sexual Violence).

Consequences to health as a result of IPV were defined as: Consequences in usual activities ("Yes" or "No," also applied to the following variables); Physical consequences (bruises, cuts, fractures, burns, or other physical injuries); Psychological consequences (fear, sadness, discouragement, difficulty sleeping, anxiety, depression or other psychological consequences); Sexually transmitted infections or unwanted pregnancy resulting from sexual violence;

Health care, due to the consequences of IPV on health were assessed via Search for health care "Yes" or "No," also applied to the following variables); Receiving health care; Location of health care (Private; SUS Primary or Secondary Health Care; SUS Tertiary Health Care; Other locations); Hospitalization for 24 hours or more. The respondent had to answer this block of variables considering the most serious violence suffered.

\section{Sociodemographic characteristics and self-perception of health}

Potentially predictors of IPV were selected and organized, according to the contextual dimensions of the analysis, in:

- Sociodemographic characteristics: Age group (18 to 24 years old; 25 to 39 years old; 40 to 59 years old); Race/Color (White; Non-white); Living with a spouse or partner; Marital status (Married;

Divorced/Separated/Widow; Single); Education (No education/Incomplete Elementary school (0 to 8 years 
of study); Complete Elementary/Incomplete High School (9 to 11 years of study); Complete High School/Incomplete Higher Education (12 to 14 years of study); Complete Higher Education/Post-Graduation (>15 years of study)); Income (up to 1 Brazilian Minimum Wage (SM); from 1 to 3 SM; from 3 to 5 SM; above 5 SM). In 2019, one SM was R\$ 998 (Brazilian Real), which was equivalent to approximately US\$250.

- Self-perceived health status: Self-rated health (Good/Very Good; Fair; Bad/Very Bad); Chest pain or discomfort (variable dichotomized into "Yes" or "No," applied to the other variables of the dimension); Sleep problems; Eating problems; Tiredness; Disinterest/Absence of pleasure; Concentration problems; Slowness or agitation; Depressive feeling; Feeling of failure; Suicidal thinking.

\section{Data analysis}

Prevalences of total IPV and IPV types, health care aspects resulting from IPV, and their respective 95\% confidence intervals were calculated. Logistic regression models were performed to estimate the crude and adjusted odds ratios (OR) for the association of sociodemographic variables and self-perceived health status with IPV. All statistical analyzes were performed using the Stata ${ }^{\circledR} 16.1$ software and, due to complex sampling, the sampling plan design was incorporated to correct point and variance estimates through the survey module. The data set and the questionnaire applied are available on the PNS 2019 website through the Oswaldo Cruz Foundation (Fiocruz) (www.pns.fiocruz.br).

\section{Ethical considerations}

This study was approved by the National Research Ethics Commission (CONEP) of the Brazilian Ministry of Health (http://conselho.saude.gov.br/comissoes-cns/conep/). All methods were conducted in accordance with national and international ethical guidelines and regulations. The informed consent was obtained from all participants. The study was approved under number 3,529,376 (August 23, 2019), and complied with Resolution n $196 / 96$ of the Brazilian National Health Council (CNS), guaranteeing voluntariness, anonymity and the possibility of giving up.

\section{Results}

Table 1 shows the sociodemographic characteristics and the health status perceived by the 34,334 women interviewed according to IPV. Women who experienced IPV were more likely to be adults aged 25 to 39 years, living with a partner and reporting being single, having completed high school or incomplete higher education, and earning up to $1 \mathrm{SM}$ than women who did not experience IPV. 
Table 1

Sociodemographic and perceived health status characteristics of women according to exposure to IPV ( $n=$ 34,334).

\begin{tabular}{|c|c|c|c|c|c|c|}
\hline \multirow{3}{*}{ Variables } & \multicolumn{6}{|c|}{ Suffered IPV* } \\
\hline & \multicolumn{3}{|l|}{ Yes } & \multicolumn{3}{|l|}{ No } \\
\hline & $\mathbf{n}$ & $\mathbf{w} \%$ & $\begin{array}{l}95 \% \\
\mathrm{Cl}\end{array}$ & $\mathbf{n}$ & w\% & $\begin{array}{l}95 \% \\
\mathrm{Cl}\end{array}$ \\
\hline \multicolumn{7}{|l|}{ Age } \\
\hline 18 to 24 years old & 416 & 19.9 & $\begin{array}{l}{[16.7 ;} \\
23.6]\end{array}$ & 3,865 & 16.6 & $\begin{array}{l}{[16.3 ;} \\
16.9]\end{array}$ \\
\hline 25 to 39 years old & 1,197 & 43.2 & $\begin{array}{l}{[39.9 ;} \\
46.5]\end{array}$ & 1,949 & 36.4 & $\begin{array}{l}{[36.2 ;} \\
36.7]\end{array}$ \\
\hline 40 to 59 years old & 1,004 & 36.9 & $\begin{array}{l}{[33.6 ;} \\
40.4]\end{array}$ & 15,903 & 46.9 & $\begin{array}{l}{[46.6 ;} \\
47.2]\end{array}$ \\
\hline Age (years) - Mean & 35.9 & & $\begin{array}{l}{[35.0 ;} \\
36.7]\end{array}$ & 38.5 & & $\begin{array}{l}{[38.4 ;} \\
38.6]\end{array}$ \\
\hline \multicolumn{7}{|l|}{ Race/Color } \\
\hline White & 804 & 39.8 & $\begin{array}{l}{[36.0 ;} \\
43.7]\end{array}$ & 10,943 & 42.1 & $\begin{array}{l}{[41.1 ;} \\
43.0]\end{array}$ \\
\hline Non-white & 1,813 & 60.2 & $\begin{array}{l}{[56.3 ;} \\
63.9]\end{array}$ & 20,772 & 57.9 & $\begin{array}{l}{[56.9 ;} \\
58.9]\end{array}$ \\
\hline \multicolumn{7}{|l|}{ Do you have a spouse or partner you live with } \\
\hline No & 1,359 & 42.8 & $\begin{array}{l}{[39.2 ;} \\
46.4]\end{array}$ & 13,720 & 39.4 & $\begin{array}{l}{[38.6 ;} \\
40.3]\end{array}$ \\
\hline Yes & 1,258 & 57.2 & $\begin{array}{l}{[53.6 ;} \\
60.8]\end{array}$ & 17,997 & 60.6 & $\begin{array}{l}\text { [59.7; } \\
61.4]\end{array}$ \\
\hline \multicolumn{7}{|l|}{ Marital status } \\
\hline Married & 705 & 33.5 & $\begin{array}{l}\text { [29.3; } \\
38.0]\end{array}$ & 11,664 & 41.3 & $\begin{array}{l}{[40.4 ;} \\
42.2]\end{array}$ \\
\hline Divorced/ Separated/Widowed & 385 & 12.3 & $\begin{array}{l}{[10.3 ;} \\
14.5]\end{array}$ & 4,113 & 11.1 & $\begin{array}{l}{[10.6 ;} \\
11.7]\end{array}$ \\
\hline Single & 1,527 & 54.2 & $\begin{array}{l}{[49.9 ;} \\
58.5]\end{array}$ & 15,940 & 47.5 & $\begin{array}{l}{[46.6 ;} \\
48.4]\end{array}$ \\
\hline \multicolumn{7}{|l|}{ Education (Years of Study) } \\
\hline $\begin{array}{l}\text { No Education/Elementary School incomplete (0 to } \\
8 \text { years) }\end{array}$ & 756 & 28.2 & $\begin{array}{l}{[24.9 ;} \\
31.8]\end{array}$ & 8,904 & 24.8 & $\begin{array}{l}{[23.9 ;} \\
25.7]\end{array}$ \\
\hline
\end{tabular}

n: sample size; w\%: weighted percentages; Cl: confidence intervals; *IPV: Intimate Partner Violence 


\begin{tabular}{|c|c|c|c|c|c|c|}
\hline \multirow[b]{2}{*}{$\begin{array}{l}\text { Complete Elementary/Incomplete Secondary } \\
\text { School (9 to } 11 \text { years) }\end{array}$} & \multicolumn{6}{|c|}{ Suffered IPV* } \\
\hline & 441 & 15.8 & $\begin{array}{l}{[13.35 ;} \\
18.5]\end{array}$ & 4,503 & 14.4 & $\begin{array}{l}{[13.7} \\
15.1]\end{array}$ \\
\hline $\begin{array}{l}\text { Complete Secondary School/Incomplete Higher } \\
\text { Education ( } 12 \text { to } 14 \text { years old })\end{array}$ & 996 & 38.8 & $\begin{array}{l}{[34.9 ;} \\
42.9]\end{array}$ & 12,043 & 41.4 & $\begin{array}{l}{[40.5 ;} \\
42.4]\end{array}$ \\
\hline $\begin{array}{l}\text { Complete Higher Education/Postgraduation (>15 } \\
\text { years) }\end{array}$ & 424 & 17.2 & $\begin{array}{l}{[14.3 ;} \\
30.4]\end{array}$ & 6,267 & 19.4 & $\begin{array}{l}{[18.5 ;} \\
20.3]\end{array}$ \\
\hline \multicolumn{7}{|l|}{ Income } \\
\hline Up to $1 \mathrm{SM}$ & 763 & 49.9 & $\begin{array}{l}{[44.7 ;} \\
55.0]\end{array}$ & 7,486 & 39.7 & $\begin{array}{l}{[38.5 ;} \\
40.9]\end{array}$ \\
\hline From 1 to $3 \mathrm{SM}$ & 677 & 40.9 & $\begin{array}{l}{[36.1 ;} \\
45.8]\end{array}$ & 7,992 & 45.8 & $\begin{array}{l}{[44.5 ;} \\
47.0]\end{array}$ \\
\hline From 3 to $5 \mathrm{SM}$ & 97 & 4.9 & $\begin{array}{l}{[3.4 ;} \\
7.0]\end{array}$ & 1,587 & 8.0 & $\begin{array}{l}{[7.4 ;} \\
8.7]\end{array}$ \\
\hline Above 5 SM & 87 & 4.3 & $\begin{array}{l}{[3.0 ;} \\
6.1]\end{array}$ & 1,475 & 6.5 & $\begin{array}{l}{[5.9} \\
7.1]\end{array}$ \\
\hline \multicolumn{7}{|l|}{ Perception of health status } \\
\hline \multicolumn{7}{|l|}{ Health self-assessment } \\
\hline Good/Very good & 1,483 & 60.4 & $\begin{array}{l}{[57.0} \\
63.7]\end{array}$ & 20,489 & 68.1 & $\begin{array}{l}{[67.2 ;} \\
69.0]\end{array}$ \\
\hline Regular & 914 & 31.4 & $\begin{array}{l}{[28.2 ;} \\
34.8]\end{array}$ & 9,346 & 26.8 & $\begin{array}{l}{[26 ;} \\
27.6]\end{array}$ \\
\hline Bad/Very bad & 220 & 8.1 & [6.1; & 1,882 & 5.1 & $\begin{array}{l}{[4.7} \\
5.5]\end{array}$ \\
\hline Chest pain or discomfort & 603 & 22.3 & $\begin{array}{l}{[19.7} \\
25.2]\end{array}$ & 4,459 & 13.7 & [13.1; \\
\hline Sleep problems & 1,477 & 54.7 & $\begin{array}{l}{[50.7 ;} \\
58.6]\end{array}$ & 12,629 & 39.2 & $\begin{array}{l}{[38.2 ;} \\
40.2]\end{array}$ \\
\hline Eating problems & 1,157 & 46.1 & $\begin{array}{l}{[41.8} \\
50.5]\end{array}$ & 8,222 & 26.1 & $\begin{array}{l}{[25.3 ;} \\
27.0]\end{array}$ \\
\hline Tiredness & 1,648 & 64.1 & $\begin{array}{l}{[60.2 ;} \\
67.8]\end{array}$ & 14,175 & 45.2 & $\begin{array}{l}{[44.2 ;} \\
46.2]\end{array}$ \\
\hline Disinterest/Absence of pleasure & 1,461 & 58.0 & $\begin{array}{l}{[54.6 ;} \\
61.4]\end{array}$ & 11,135 & 35.0 & $\begin{array}{l}{[34.0 ;} \\
36.0]\end{array}$ \\
\hline Concentration problems & 1,112 & 45.0 & $\begin{array}{l}{[40.9} \\
49.1]\end{array}$ & 7,996 & 25.6 & $\begin{array}{l}{[24.7 ;} \\
26.5]\end{array}$ \\
\hline
\end{tabular}

n: sample size; w\%: weighted percentages; Cl: confidence intervals; *IPV: Intimate Partner Violence 


\begin{tabular}{|c|c|c|c|c|c|c|}
\hline \multirow[b]{2}{*}{ Slowness or agitation } & \multicolumn{6}{|c|}{ Suffered IPV* } \\
\hline & 961 & 37.3 & $\begin{array}{l}{[34.1 ;} \\
40.5]\end{array}$ & 6,866 & 21.3 & $\begin{array}{l}\text { [20.5; } \\
22.1]\end{array}$ \\
\hline Depressive feeling & 1,549 & 58.5 & $\begin{array}{l}{[54.3 ;} \\
62.6]\end{array}$ & 10,517 & 32.9 & $\begin{array}{l}\text { [32.0; } \\
33.8]\end{array}$ \\
\hline Feeling of failure & 1,122 & 44.8 & $\begin{array}{l}{[40.7 ;} \\
49.0]\end{array}$ & 6,394 & 20.4 & $\begin{array}{l}{[19.6 ;} \\
21.2]\end{array}$ \\
\hline Suicidal thinking & 480 & 20.2 & $\begin{array}{l}{[17.2 ;} \\
23.6]\end{array}$ & 1,810 & 5.4 & $\begin{array}{l}{[5.0 ;} \\
5.9]\end{array}$ \\
\hline
\end{tabular}

Compared with women who did not suffer IPV, women who suffered IPV reported a worse perception of their health status. For example, women who experienced IPV (compared to women who did not experience IPV) reported, respectively, a higher proportion of bad or very bad health status ( $8.1 \%$ vs $5.1 \%)$, tiredness (64.1\% vs $45.2 \%$ ), feeling depressed (58.5\%) vs $32.0 \%$ ), disinterest/absence of pleasure (58.0\% vs $35.0 \%$ ), sleeping problems ( $54.7 \%$ vs $39.2 \%)$ and eating problems (46.1\% vs $26.1 \%$ ) (Table 1$)$.

The prevalence of IPV in Brazilian women in the last 12 months was 7.6\% (95\% Cl: [7.0; 8.2]) (Table 2). Regarding the types of IPV, the most prevalent were verbal violence $(6.2 \% ; 95 \% \mathrm{Cl}$ : [5.7; 6.8]), followed by psychological violence $(4.3 \%, 95 \% \mathrm{Cl}$ : [3.8; 4.8]), threat $(2.8 \%, 95 \% \mathrm{Cl}:[2.5 ; 3.1])$ and physical violence $(2.7 \%$, $95 \% \mathrm{Cl}:[2.4 ; 3.2])$. Although less common, the prevalence of economic violence $(1.5 \%, 95 \% \mathrm{Cl}$ : $[1.3 ; 1.8]$ and digital violence $(1.0 \%, 95 \% \mathrm{Cl}$ : $[0.8 ; 1.2])$ perpetrated by partners were also found. The least prevalent type was sexual violence $(0.7 \%, 95 \% \mathrm{Cl}$ : $[0.5 ; 0.9])$. Table 2 also expresses the estimated number of adult Brazilian women who suffered IPV and its subtypes in the last 12 months, with a total of 4,949,780 women. 
Table 2

Prevalence of types of violence committed against women aged 18 to 59, perpetrated by an intimate partner in the last 12 months $(n=34,334)$.

\begin{tabular}{|lccc|}
\hline & weighted $\mathbf{n}$ & w\% & $95 \% \mathbf{C l}$ \\
\hline Any IPV & $4,949,780$ & 7.6 & {$[7.0 ; 8.2]$} \\
\hline Verbal violence & $4,053,244$ & 6.2 & {$[5.7 ; 6.8]$} \\
\hline Psychological violence & $2,814,532$ & 4.3 & {$[3.8 ; 4.8]$} \\
\hline Threat & $1,824,633$ & 2.8 & {$[2.5 ; 3.1]$} \\
\hline Physical violence & $1,793,997$ & 2.7 & {$[2.4 ; 3.2]$} \\
\hline Economic/Property Violence & $1,011,088$ & 1.5 & {$[1.3 ; 1.8]$} \\
\hline Digital/Technological Violence & 662,248 & 1.0 & {$[0.8 ; 1.2]$} \\
\hline Sexual violence & 441,885 & 0.7 & {$[0.5 ; 0.9]$} \\
\hline w\%: weighted percentages; Cl: confidence intervals; IPV: intimate partner violence. \\
\hline
\end{tabular}

Regarding the health consequences of IPV (Table 3), 69.1\% (95\% Cl: [66.1; 72.0]) of women who suffered IPV reported psychological consequences, such as fear, discouragement, difficulty sleeping, anxiety, depression, or other psychological consequences. Among those who suffered sexual violence, $8.1 \%$ (95\% Cl: $[6.0 ; 10.9])$ reported having contracted a sexually transmitted infection or unwanted pregnancy due to sexual abuse.

Among women who suffered health consequences, $13.9 \%$ (95\% Cl: $[12.0 ; 16.2])$ sought health care, of which $88.8 \%$ (95\% Cl: [84.9; 91.8]) were attended. The most sought after place was the primary health care (PHC) or secondary care of the Unified Health System (SUS) $(41.9 \%, 95 \%$ Cl: [37.8; 46.1]), which along with public hospitals or public emergency units $(21.4 \%, 95 \% \mathrm{Cl}$ : $117.6 ; 25.8])$ accounted for more than $63 \%$ of the assistance provided to victims in public health, in contrast to a minority that received care in private health services $(26.9 \%, 95 \% \mathrm{Cl}:[23.4 ; 30.8])$. 
Table 3

Prevalence of health consequences and aspects of health care for women as consequence of IPV.

$\begin{array}{lllll}\text { Variables } & n & w \% & 95 \% \mathrm{Cl}\end{array}$

\section{Consequences of IPV for health}

Failed to perform usual activities

\begin{tabular}{lll}
$593 / 2,617$ & 22.2 & {$[19.8 ; 24.8]$} \\
\hline $430 / 2,617$ & 16.3 & {$[13.2 ; 20.0]$} \\
\hline $1,790 / 2,617$ & 69.1 & {$[66.1 ; 72.0]$}
\end{tabular}

Psychological consequences $^{c}$

$1,790 / 2,617$

\section{Health consequences of sexual violence}

Sexually transmitted infection or unwanted pregnancy $\quad 23 / 227 \quad 8.1 \quad[6.0 ; 10.9]$

\section{Health care due to IPV}

\begin{tabular}{llll} 
Search for health care & $374 / 2,617$ & 13.9 & {$[12.0 ; 16.2]$} \\
\hline Health care assistance & $338 / 374$ & 88.8 & {$[84.9 ; 91.8]$} \\
\hline Location of health care service & & & \\
\hline Private & $93 / 338$ & 26.9 & {$[23.4 ; 30.8]$} \\
\hline SUS Primary/Secondary Care & $132 / 338$ & 41.9 & {$[37.8 ; 46.1]$} \\
\hline SUS Tertiary Care & $82 / 338$ & 21.4 & {$[17.6 ; 25.8]$} \\
\hline Other places $^{d}$ & $31 / 338$ & 9.8 & {$[7.0 ; 13.5]$} \\
\hline Hospitalization for 24 hours or more $^{\text {a }}$ & $17 / 338$ & 6.2 & {$[4.5 ; 8.4]$}
\end{tabular}

n: sample size; w\%: weighted percentages; Cl: confidence intervals;

a The variables refer only to the most severe type of IPV suffered by women;

b Bruises, cuts, fractures, burns or other physical injuries;

${ }^{c}$ Fear, sadness, discouragement, difficulty sleeping, anxiety, depression or other psychological consequences;

d Pharmacy, home, place of occurrence of IPV and others.

In the multivariable logistic regression model (Table 4), women aged 18 to 24 years and 25 to 39 years had higher odds of IPV compared to women aged over 40 years $(\mathrm{OR}=1.45 ; 95 \% \mathrm{Cl}[1.01 ; 2.08]$ and $\mathrm{OR}=1.46 ; 95 \%$ $\mathrm{Cl}[1.14 ; 1.87]$, respectively). Divorced/separated/widowed and single women had higher odds of IPV than married women, $\mathrm{OR}=1.64$ (95\% $\mathrm{Cl}[1.18 ; 2.27])$ and $\mathrm{OR}=1.38$ (95\% $\mathrm{Cl}[1.00 ; 1.89])$, respectively. Women who earn up to 1 minimum wage had greater odds of IPV than women with an income above 5 minimum wages, $\mathrm{OR}=1.61(95 \% \mathrm{Cl}[0.99 ; 2.61] ; \mathrm{p}=0.029)$. Regarding the health perception variables, women who reported eating problems $(\mathrm{OR}=1.29 ; 95 \% \mathrm{Cl}[1.01 ; 1.65])$, lack of interest/absence of pleasure $(\mathrm{OR}=1.41 ; 95 \% \mathrm{Cl}[1.11$; 1.79]), depressive feeling $(\mathrm{OR}=1.39 ; 95 \% \mathrm{Cl}[1.03 ; 1.88])$, feeling of failure $(\mathrm{OR}=1.75 ; 95 \% \mathrm{Cl}[1.36 ; 2.24])$ and 
suicidal thinking $(\mathrm{OR}=1.73 ; 95 \% \mathrm{Cl}[1.25 ; 2.41])$ had higher odds of IPV than women who did not report these same perceptions. 
Table 4

Logistic regression for the association between sociodemographic characteristics and perceived health and IPV $(\mathrm{n}=34,334)$.

\begin{tabular}{|c|c|c|c|c|c|c|}
\hline \multirow[b]{2}{*}{ Variables } & \multicolumn{6}{|c|}{ Suffered Violence } \\
\hline & COR & $\begin{array}{l}95 \% \\
\mathrm{Cl}\end{array}$ & p & $\mathrm{aOR}$ & $\begin{array}{l}95 \% \\
\mathrm{Cl}\end{array}$ & p \\
\hline Age & & & $<0.001$ & & & 0.010 \\
\hline 18 to 24 & 1.52 & $\begin{array}{l}{[1.18 ;} \\
1.97]\end{array}$ & 0.001 & 1.45 & $\begin{array}{l}{[1.01 ;} \\
2.08]\end{array}$ & 0.043 \\
\hline 25 to 39 & 1.51 & $\begin{array}{l}{[1.28 ;} \\
1.76]\end{array}$ & $<0.001$ & 1.46 & $\begin{array}{l}{[1.14 ;} \\
1.87]\end{array}$ & 0.003 \\
\hline 40 to 59 & 1.00 & & & 1.00 & & \\
\hline Race & & & 0.268 & & & 0.763 \\
\hline White & 1.00 & & & 1.00 & & \\
\hline Non-white & 1.10 & $\begin{array}{l}{[0.93} \\
1.29]\end{array}$ & 0.268 & 0.96 & $\begin{array}{l}{[0.75 ;} \\
1.23]\end{array}$ & 0.763 \\
\hline Has a spouse or partner she lives with & 0.87 & $\begin{array}{l}{[0.75 ;} \\
1.02]\end{array}$ & 0.083 & 1.19 & $\begin{array}{l}{[0.93 ;} \\
1.52]\end{array}$ & 0.166 \\
\hline Marital status & & & 0.007 & & & 0.013 \\
\hline Married & 1.00 & & & 1.00 & & \\
\hline Divorced/ Separated/Widowed & 1.36 & $\begin{array}{l}{[1.05 ;} \\
1.74]\end{array}$ & 0.016 & 1.64 & $\begin{array}{l}{[1.18 ;} \\
2.27]\end{array}$ & 0.003 \\
\hline Single & 1.41 & $\begin{array}{l}{[1.13} \\
1.75]\end{array}$ & 0.002 & 1.38 & $\begin{array}{l}{[1.00 ;} \\
1.89]\end{array}$ & 0.048 \\
\hline Education (Years of Study) & & & 0.077 & & & 0.075 \\
\hline $\begin{array}{l}\text { No education/ Elementary school incomp. (0 } \\
\text { to } 8 \text { years) }\end{array}$ & 1.28 & $\begin{array}{l}{[1.02 ;} \\
1.61]\end{array}$ & 0.033 & 1.00 & $\begin{array}{l}{[0.70} \\
1.45]\end{array}$ & 0.973 \\
\hline $\begin{array}{l}\text { Elementary/Secondary incomplete (9 to } 11 \\
\text { years) }\end{array}$ & 1.24 & $\begin{array}{l}{[0.95 ;} \\
1.62]\end{array}$ & 0.117 & 0.87 & $\begin{array}{l}{[0.61 ;} \\
1.26]\end{array}$ & 0.472 \\
\hline $\begin{array}{l}\text { Secondary/Incomplete Higher education ( } 12 \\
\text { to } 14 \text { years) }\end{array}$ & 1.06 & $\begin{array}{l}{[0.82 ;} \\
1.36]\end{array}$ & 0.663 & 0.75 & $\begin{array}{l}{[0.56 ;} \\
0.99]\end{array}$ & 0.046 \\
\hline $\begin{array}{l}\text { Complete higher education/Postgraduation } \\
\text { (>15 years) }\end{array}$ & 1.00 & & & 1.00 & & \\
\hline Income & & & $<0.001$ & & & 0.029 \\
\hline
\end{tabular}

cOR: crude odds ratio; aOR: adjusted odds ratio; Cl: confidence intervals; Bold font indicates Wald test.

* Comparison refereed to yes vs. no (reference group). 


\begin{tabular}{|c|c|c|c|c|c|c|}
\hline \multirow[b]{2}{*}{ Up to $1 \mathrm{SM}$} & \multicolumn{6}{|c|}{ Suffered Violence } \\
\hline & 1.88 & $\begin{array}{l}{[1.27 ;} \\
2.80]\end{array}$ & 0.002 & 1.61 & $\begin{array}{l}{[0.99 ;} \\
2.61]\end{array}$ & 0.053 \\
\hline From 1 to $3 \mathrm{SM}$ & 1.34 & $\begin{array}{l}{[0.91 ;} \\
1.97]\end{array}$ & 0.137 & 1.23 & $\begin{array}{l}{[0.79 ;} \\
1.90]\end{array}$ & 0.352 \\
\hline From 3 to $5 \mathrm{SM}$ & 0.92 & $\begin{array}{l}{[0.60} \\
1.42]\end{array}$ & 0.714 & 0.85 & $\begin{array}{l}\text { [0.56; } \\
1.29]\end{array}$ & 0.448 \\
\hline Above 5 SM & 1.00 & & & 1.00 & & \\
\hline \multicolumn{7}{|l|}{ PERCEPTION OF HEALTH } \\
\hline Health self-assessment & & & $<0.001$ & & & 0.112 \\
\hline Good/Very good & 1.00 & & & 1.00 & & \\
\hline Regular & 1.32 & $\begin{array}{l}{[1.12 ;} \\
1.55]\end{array}$ & 0.001 & 0.87 & $\begin{array}{l}{[0.68 ;} \\
1.12]\end{array}$ & 0.288 \\
\hline Bad/Very bad & 1.81 & $\begin{array}{l}{[1.31 ;} \\
2.50]\end{array}$ & $<0.001$ & 0.62 & $\begin{array}{l}{[0.39 ;} \\
0.97]\end{array}$ & 0.038 \\
\hline Chest pain or discomfort* & 1.80 & $\begin{array}{l}{[1.52 ;} \\
2.13]\end{array}$ & $<0.001$ & 0.98 & $\begin{array}{l}\text { [0.77; } \\
1.27]\end{array}$ & 0.911 \\
\hline Sleep problems* & 1.87 & $\begin{array}{l}{[1.59 ;} \\
2.22]\end{array}$ & $<0.001$ & 1.04 & $\begin{array}{l}{[0.83 ;} \\
1.30]\end{array}$ & 0.721 \\
\hline Eating problems* & 2.42 & $\begin{array}{l}{[2.02 ;} \\
2.89]\end{array}$ & $<0.001$ & 1.29 & $\begin{array}{l}{[1.01 ;} \\
1.65]\end{array}$ & 0.042 \\
\hline Felt tired* & 2.16 & $\begin{array}{l}{[1.84 ;} \\
2.55]\end{array}$ & $<0.001$ & 1.01 & $\begin{array}{l}{[0.81 ;} \\
1.26]\end{array}$ & 0.903 \\
\hline Disinterest/Absence Pleasure* & 2.57 & $\begin{array}{l}{[2.23} \\
2.96]\end{array}$ & $<0.001$ & 1.41 & $\begin{array}{l}{[1.11 ;} \\
1.79]\end{array}$ & 0.005 \\
\hline Concentration problems* & 2.38 & $\begin{array}{l}{[2.00 ;} \\
2.83]\end{array}$ & $<0.001$ & 1.13 & $\begin{array}{l}{[0.88 ;} \\
1.45]\end{array}$ & 0.332 \\
\hline Slowness or agitation* & 2.20 & $\begin{array}{l}{[1.90 ;} \\
2.54]\end{array}$ & $<0.001$ & 1.09 & $\begin{array}{l}{[0.83 ;} \\
1.42]\end{array}$ & 0.538 \\
\hline Felt depressed* & 2.88 & $\begin{array}{l}{[2.41 ;} \\
3.43]\end{array}$ & $<0.001$ & 1.39 & $\begin{array}{l}{[1.03 ;} \\
1.88]\end{array}$ & 0.031 \\
\hline Feeling of failure* & 3.17 & $\begin{array}{l}{[2.66 ;} \\
3.77]\end{array}$ & $<0.001$ & 1.75 & $\begin{array}{l}{[1.36 ;} \\
2.24]\end{array}$ & $<0.001$ \\
\hline Suicidal thinking* & 4.42 & $\begin{array}{l}\text { [3.56; } \\
5.51]\end{array}$ & $<0.001$ & 1.73 & $\begin{array}{l}{[1.25 ;} \\
2.41]\end{array}$ & 0.001 \\
\hline
\end{tabular}




\section{Discussion}

Our study including a representative sample of the Brazilian population estimated that $7.6 \%$ of adult Brazilian women reported to have suffered IPV in the last 12 months. IPV was more prevalent in younger women (up to 39 years old), divorced or single, low-income, with more eating and mental health problems, such as anhedonia, feeling of disinterest in life, despair, failure, and suicidal ideation. The study also showed that, as a result of IPV, many women reported health consequences and sought care in health services, especially in the context of public health.

The prevalence of IPV (7.6\%) found in our study corroborates the most recent estimates by the $\mathrm{WHO}^{12}$ and preliminary analyses by the Brazilian $\mathrm{PNS}^{17}$. On the other hand, it signals a possible reduction in IPV prevalence compared to the WHO multicentric study also carried out in Brazil about two decades ago ${ }^{10,23}$. In that study, the prevalence of IPV in the last 12 months for Brazilian women aged 15 to 49 ranged from 18.7-24.2\% for psychological violence; $8.3-12.9 \%$ for physical violence; and $2.8-5.6 \%$ for sexual violence ${ }^{23}$. Such differences could result from methodological differences between the studies. Another hypothesis is that, as Brazil has been making efforts to prevent IPV over the last 15 years, with the approval of specific laws to prevent and restrain violence against women ${ }^{24-26}$, such differences may be a consequence of these laws. Brazilian legislation has expanded the scope, contemplating the classification of violence beyond physical and sexual violence, including psychological, economic, moral, verbal, digital (virtual) violence, threats, among others ${ }^{24,26-28}$.

The most prevalent types of IPV in our study were verbal and psychological violence, the latter being typified in a specific law in $2021^{26}$, given its high prevalence and challenging evidence, different from physical and sexual violence, which leave visible marks on women's bodies. Our results, therefore, support the need for this type of legislation, which is more specific for each type of violence.

Our study also reveals unprecedented data on the prevalence of types of IPV that are less described in the literature in population-based studies, such as economic and digital violence, which are also typified in Brazilian legislation ${ }^{24,27}$. Although physical violence has been less prevalent than other types of violence, perhaps because it leaves "evidence" that more easily incriminates offenders, the negative impacts that non-physical abuse has on women's health and well-being should be highlighted ${ }^{29,30}$. For instance, a major source of stress for victims is economic violence, one of the least studied forms of violence and often included in emotional or psychological abuse, encompasses controlling and limiting access to financial resources, denying access to needs (e.g., food, clothing, medication), tracking the use of money and also withholding or hiding the money earned together ${ }^{30}$. Digital (or technological) intimate partner violence provides a quick and easy method of harassing women, using social media, GPS tracking, constant texting, or other forms of intimidation, embarrassment, and control, and it has been described as more frequent due to the COVID-19 pandemic ${ }^{31}$. Therefore, digital violence is a type of violence that deserves to be further studied through comparative analyzes before and after the pandemic, and our study portrays the prevalence in the year just before the pandemic. 
Our study also brings unprecedented nationwide findings on the impacts of IPV on the health of Brazilian women. Two out of three women who suffered IPV in the past 12 months reported experiencing psychological consequences from the abuse, while one in six women reported physical consequences such as bruises, cuts, fractures, burns, or other physical injuries. Sexual violence practiced by intimate partners, including forced sexual intercourse, also had consequences for sexual and reproductive health, such as sexually transmitted infections and unwanted pregnancies for one in every twelve Brazilian women who suffered this type of abuse. These data corroborate the international literature on the impacts of IPV on women's health ${ }^{6,32-34}$. Consequently, almost one in four women stopped performing their usual activities as a result of IPV. International studies indicate that IPV can increase the chances of women experiencing difficulties with daily activities by up to $60 \%{ }^{9}$. That reinforces the need for public policies and specific interventions aimed at health services, including the training of health professionals to care for these women who seek health services.

Our study also identified predictor variables for IPV in women. In the adjusted model, younger women were almost $50 \%$ more likely to suffer IPV compared to older ones (over 39 years old), as it converges with the international literature that postulates that the younger ages are strongly associated with increased risk of IPV in the previous year ${ }^{35,36}$. Possible justifications for this include that younger women are more likely to live in relationships of greater dependence on their partner (financial and related to childcare); in comparison, older women tend to have greater financial and social autonomy, providing opportunities to leave abusive relationships (17). Odds of IPV are also higher for unmarried women, including single (with $38 \%$ higher odds) and divorced/separated/widowed women (64\% higher odds) than married women. These findings are aligned with results from other countries ${ }^{36}$, in which women who lived with a partner without being formally married had an increased risk of IPV 35 .

On the other hand, unlike studies from other countries ${ }^{35-38}$, the results of our adjusted model did not demonstrate that more educated women are less exposed to IPV. In studies of other low- and middle-income countries, in addition to women with less education, lower partner education was also associated with IPV against women 39,40 , but the Brazilian PNS did not collect data on the education of partners, which limited our analyses.

Regarding self-rated health, women who reported IPV were more likely to report eating problems, lack of interest/lack of pleasure, depressive feelings, feeling of failure, and suicidal thoughts. The findings converge with international studies, showing that women who suffer IPV reported more self-reported health problems, including eating disorders and implications for mental health ${ }^{6,9,33,41}$.

As a result of abuse, women facing IPV use health services more frequently than other women ${ }^{4,6}$. Regarding our findings on the use of health services, only one in seven Brazilian women sought health care due to IPV. On the other hand, of those who sought care, the vast majority (nine out of ten) received care, mostly in public health services, with emphasis on primary or secondary health care services. This result reinforces the importance of universal, free and accessible public health services and the important role of primary health care for survivors ${ }^{42,43}$. Women who have suffered violence can seek health services before seeking 
police or social services. If violence is identified, survivors can receive care that increases their safety and improves their health ${ }^{6,21}$. Therefore, health services are privileged spaces to offer care to these women and prevent the escalation of violence ${ }^{42,43}$. Health professionals are considered key in supporting women experiencing violence ${ }^{44}$, playing a decisive role in identifying IPV and supporting them ${ }^{45}$. However, such identification is not always easy. Many women are afraid, ashamed, or lack confidence in revealing their IPV situation to professionals, even when questioned 46,47 ; this causes IPV to be underrecognized by health providers $^{32}$. Therefore, professionals, especially those working in primary health care, need to be aware of the profile of women most exposed to IPV and the signs and symptoms shown by them so that they can provide adequate care. According to our study, these signs and symptoms may include eating problems and mental health issues, like lack of interest, absence of pleasure, depressive feeling, a sense of failure, and suicidal thinking.

One of the limitations derived from the study design is that it is not possible to establish whether the exposure to violence occurred before or after the self-perceived health reports. Previous studies and theoretical reasoning suggest that health problems perceived by women are mainly the result of abuse and not its precursors ${ }^{6,9}$. It is also possible that the results have a bias in IPV classification since many may not remember or even consider insults, threats, or humiliation suffered as violence. Due to the social stigma associated with disclosing victimization ${ }^{32}$, feelings such as fear, shame, or repressed memories can decrease the probability of disclosing and discussing violence with a stranger, especially sexual and intimate violence ${ }^{33}$. Similar to national health surveys from other countries ${ }^{48}$, due to privacy issues and the seriousness of violence in the domestic environment, a possible limitation is that many of the respondents were not willing to report the IPV they suffered. The sample also excluded homeless populations, villagers, and institutionalized women in places such as jails or shelters, which may contribute to an underestimation.

\section{Conclusions}

The findings of our study reveal that women who suffered IPV reported health problems more frequently, such as physical and psychological injuries and consequences for sexual and reproductive health. Although not all women seek help from health services due to IPV, most who do, seek support from the SUS (Brazilian public health system), especially in primary or secondary health care services. Younger, divorced or single, low-income women with eating and mental health problems were more likely to experience IPV. Thus, the training of health professionals to identify and care for women who suffer from IPV is an important step so they can be allies in the prevention and reduction of violence against women.

\section{Declarations}

\section{Ethics approval and consent to participate:}

This study was approved by the National Research Ethics Commission (CONEP) of the Brazilian Ministry of Health (http://conselho.saude.gov.br/comissoes-cns/conep/). All methods were conducted in accordance with national and international ethical guidelines and regulations. The informed consent was obtained from 
all participants. The study was approved under number 3,529,376 (August 23, 2019), and complied with Resolution $n^{0} 196 / 96$ of the Brazilian National Health Council (CNS), guaranteeing voluntariness, anonymity and the possibility of giving up.

\section{Consent for publication:}

NA

\section{Availability of data and materials:}

the dataset utilized in this study is publicly available for download at the website of the "Fundação Oswaldo Cruz" - FIOCRUZ [https://www.pns.icict.fiocruz.br/bases-de-dados/]. The datasets analysed during the current study are available in the Google Drive Folder repository,

[https://drive.google.com/drive/folders/1IErJnxJXZ1sPsUx9bWhF5ULsOM6PniTP?usp=sharing]. It is also available from the corresponding author on request.

\section{Competing interests:}

The authors declare no conflict of interest.

\section{Funding:}

none

\section{Authors' contributions:}

MS conceived the study, collaborated with the analyses, drafted the introduction and discussion, and critically revised the text; FS led the analysis, drafted the results and critically revised the text; RPJr collaborated in the analyses, drafted the abstract and methods, and critically revised the text; JV collaborated with the analyses, drafted the conclusion and critically revised the text; SA conceived the statistical design, supervised the analyzes and critically revised the text; LR supervised the analysis and writing of the article and critically reviewed the text; ZS supervised the analysis and writing of the article, structured the discussion axes and critically revised the text. All authors read and revised the text incorporating significant contributions to the manuscript. The team met weekly for 3 months to discuss the methodological design, conduct the analyses and discuss the results. All approved the final version of the manuscript.

\section{Acknowledgements:}


not applicable

\section{Authors' information (optional):}

not applicable

\section{References}

1. World Health Organization. Global and regional estimates of violence against women: prevalence and health effects of intimate partner violence and non-partner sexual violence. Geneva, Switzerland; 2013. Available from: https://www.who.int/publications/i/item/9789241564625

2. World Health Organization (WHO). Intimate Partner Violence [Internet]. Understanding and addressing violence against women. Geneva, Switzerland; 2012. Available from:

http://apps.who.int/iris/bitstream/handle/10665/77432/WHO_RHR_12.36_eng.pdf?sequence=1

3. Guruge S. Intimate partner violence: a global health perspective. The Canadian Journal of Nursing Research = Revue Canadienne de Recherche en Sciences Infirmieres. 2012 Dec;44(4):36-54.

4. Black MC. Intimate Partner Violence and Adverse Health Consequences: Implications for Clinicians. American Journal of Lifestyle Medicine [Internet]. 2011;5(5):428-39. Available from:

http://journals.sagepub.com/doi/abs/10.1177/1559827611410265\#articleCitationDownloadContainer

5. Dutton MA, Green BL, Kaltman SI, Roesch DM, Zeffiro TA, Krause ED. Intimate partner violence, PTSD, and adverse health outcomes. Journal of Interpersonal Violence [Internet]. 2006;21(7):955-68.

Available from: https://www.ncbi.nlm.nih.gov/pubmed/16731994

6. Campbell JC. Health consequences of intimate partner violence. Lancet [Internet]. 2002;359(9314):1331-6. Available from: https://www.ncbi.nlm.nih.gov/pubmed/11965295

7. Cruz MS, Irffi G. Qual o efeito da violência contra a mulher brasileira na autopercepção da saúde? Ciência \& Saúde Coletiva [Internet]. 2019 Jul 22 [cited 2021 Nov 12];24(7):2531-42. Available from: http://www.scielo.br/scielo.php?script=sci_arttext\&pid=S1413-81232019000702531\&tlng=pt

8. de Aquino NMR, Sun SY, de Oliveira EM, Martins M da G, da Silva J de F, Mattar R. Sexual violence and its association with health selfperception among pregnant women. Revista de Saude Publica. 2009;43(6):1-6.

9. Ellsberg M, Jansen HA, Heise L, Watts CH, Garcia-Moreno C. Intimate partner violence and women's physical and mental health in the WHO multi-country study on women's health and domestic violence: an observational study. The Lancet [Internet]. 2008;371(9619):1165-72. Available from: http://www.ncbi.nlm.nih.gov/pubmed/18395577

10. Garcia-Moreno C, Jansen HA, Ellsberg M, Heise L, Watts $\mathrm{CH}$, Team WHOMS on WH, et al. Prevalence of intimate partner violence: findings from the WHO multi-country study on women's health and domestic violence. Lancet [Internet]. 2006;368(9543):1260-9. Available from:

http://www.ncbi.nlm.nih.gov/pubmed/17027732 
11. Coll CVN, Ewerling F, García-Moreno C, Hellwig F, Barros AJD. Intimate partner violence in 46 low-income and middle-income countries: an appraisal of the most vulnerable groups of women using national health surveys. BMJ Global Health [Internet]. 2020 Jan 1;5(1):e002208. Available from: http://gh.bmj.com/content/5/1/e002208.abstract

12. World Health Organization. Violence against women prevalence estimates, 2018 [Internet]. Global, regional and national prevalence estimates for intimate partner violence against women and global and regional prevalence estimates for non-partner sexual violence against women. Geneva, Switzerland; 2021. Available from: papers2://publication/uuid/083CEB59-0975-4621-B202C8AD43C62F34

13. Waiselfisz JJ. Mapa da Violência 2015: Homicídio de mulheres no Brasil [Internet]. Vol. 1, Flacso Brasil. 2015. Available from: http://www.mapadaviolencia.org.br/pdf2015/MapaViolencia_2015_mulheres.pdf

14. Garcia LP, de Freitas LR, da Silva GD, Höfelmann DA. [Corrected estimates of femicides in Brazil, 2009 to 2011]. Rev Panam Salud Publica [Internet]. 2015;37(4-5):251-7. Available from: https://www.ncbi.nlm.nih.gov/pubmed/26208193

15. Zara G, Gino S. Intimate Partner Violence and its Escalation Into Femicide. Frailty thy Name Is "Violence Against Women." Frontiers in psychology [Internet]. 2018 September 26;9:1777. Available from: https://pubmed.ncbi.nIm.nih.gov/30319489

16. UN. Transforming our world: the 2030 agenda for sustainable development [Internet]. 2015. Available from: https://www.un.org/pga/wp-content/uploads/sites/3/2015/08/120815_outcome-document-ofSummit-for-adoption-of-the-post-2015-development-agenda.pdf

17. Vasconcelos NM de, Martins F, Andrade D de, Gomes CS. Prevalência e fatores associados a violência por parceiro íntimo contra mulheres adultas no Brasil: Pesquisa Nacional de Saúde, 2019. Revista Brasileira de Epidemiologia. 2021;24(Suppl 2): e210020. https://doi.org/10.1590/1980549720210020.supl.2.

18. Malta DC, Szwarcwald CL, da Silva Júnior JB. First results of laboratory analysis in the national health survey. Revista Brasileira de Epidemiologia. 2019;22(Suppl 2):2-4. doi:10.1590/1980549720190001.supl.2

19. Szwarcwald CL, Malta DC, Pereira CA, Vieira MLFP, Conde WL, de Souza Júnior PRB, et al. Pesquisa nacional de saúde no Brasil: Concepção e metodologia de aplicação. Ciencia e Saude Coletiva. 2014;19(2):333-42. https://doi.org/10.1590/1413-81232014192.14072012.

20. Ellsberg M, Heise L. Researching Violence Against Women: A Practical Guide for Researchers and Activists [Internet]. Washington DC; 2005. Available from:

https://www.who.int/reproductivehealth/publications/violence/9241546476/en/

21. World Health Organization. Responding to intimate partner violence and sexual violence against women: WHO clinical and policy guidelines [Internet]. Geneva; 2013. Available from: https://www.who.int/reproductivehealth/publications/violence/9789241548595/en/

22. Stopa SR, Szwarcwald CL, Oliveira MM de, Gouvea E de CDP, Vieira MLFP, Freitas MPS de, et al. Pesquisa Nacional de Saúde 2019: histórico, métodos e perspectivas. Epidemiologia e servicos de 
saude: revista do Sistema Unico de Saude do Brasil. 2020;29(5):e2020315.

http://dx.doi.org/10.1590/s1679-49742020000500004

23. Schraiber LB, D'Oliveira AF, França-Junior I, Diniz S, Portella AP, Ludermir AB, et al. Prevalence of intimate partner violence against women in regions of Brazil. Rev Saude Publica [Internet]. 2007;41(5):797-807. Available from: http://www.ncbi.nlm.nih.gov/pubmed/17923901

24. Brazilian Federal Government. Law number 11,340/2006 [Internet]. Vol. 11340. Brasilia: Federal Government; 2006. Available from: http://www.planalto.gov.br/ccivil_03/_ato20042006/2006/lei/l11340.htm

25. Brazilian Federal Government. Law number 13,104 from 09/03/2015 [Internet]. Brasil; 2015. Available from: http://www.planalto.gov.br/ccivil_03/_ato2015-2018/2015/lei//13104.htm

26. Brazilian Federal Government. Law number 14,188, from 28/07/2021 [Internet]. Brazil; 2021 p. 1. Available from: https://www.in.gov.br/web/dou/-/lei-n-14.188-de-28-de-julho-de-2021-334902612

27. Brazilian Federal Government. Law number 13,185, from 06/11/2015 [Internet]. Brazil; 2015. Available from: http://www.planalto.gov.br/ccivil_03/_ato2015-2018/2015/lei//13185.htm

28. Política Nacional de Enfrentamento à Violência contra as Mulheres [National Policy to Combat Violence Against Women]. [Internet]. Brasilia; 2011. Available from:

https://www12.senado.leg.br/institucional/omv/entenda-a-violencia/pdfs/politica-nacional-deenfrentamento-a-violencia-contra-as-mulheres

29. Tiwari S, Gray R, Jenkinson C, Carson C. Association between spousal emotional abuse and reproductive outcomes of women in India: findings from cross-sectional analysis of the 2005-2006 National Family Health Survey. Social Psychiatry and Psychiatric Epidemiology [Internet]. 2018;53(5):509-19. Available from: http://dx.doi.org/10.1007/s00127-018-1504-3

30. Stylianou AM. Economic abuse within intimate partner violence: A review of the literature. Violence and Victims. 2018;33(1):3-22. doi:10.1891/0886-6708.VV-D-16-00112

31. Maftei A, Dănilă O. Give me your password! What are you hiding? Associated factors of intimate partner violence through technological abuse. Current Psychology. 2021; doi:10.1007/s12144-02102197-2

32. Fanslow J. Intimate partner violence and women's reproductive health. Obstetrics, Gynaecology and Reproductive Medicine [Internet]. 2017;27(5):148-57. Available from: http://dx.doi.org/10.1016/j.ogrm.2017.02.003

33. Plichta SB, Falik M. Prevalence of violence and its implications for women's health. Women's Health Issues. 2001;11(3):244-58. doi:10.1016/s1049-3867(01)00085-8

34. Loxton D, Schofield M, Hussain R. Psychological health in midlife among women who have ever lived with a violent partner or spouse. Journal of interpersonal violence [Internet]. 2006 Aug 2 [cited 2021 Nov 13];21(8):1092-107. Available from:

http://journals.sagepub.com/doi/10.1177/0886260506290290

35. Abramsky T, Watts CH, Garcia-Moreno C, Devries K, Kiss L, Ellsberg M, et al. What factors are associated with recent intimate partner violence? findings from the WHO Multi-country Study on 
women's Health and Domestic Violence. BMC Public Health [Internet]. 2011;11:109. Available from: http://www.ncbi.nlm.nih.gov/pubmed/21324186

36. Yakubovich AR, StöckI H, Murray J, Melendez-Torres GJ, Steinert JI, Glavin CEY, et al. Risk and Protective Factors for Intimate Partner Violence Against Women: Systematic Review and Meta-analyses of Prospective-Longitudinal Studies. American journal of public health [Internet]. 2018 Jul 6 [cited 2021 Nov 13];108(7):e1-11. Available from:

https://ajph.aphapublications.org/doi/10.2105/AJPH.2018.304428

37. Edeby A, San Sebastián M. Prevalence and sociogeographical inequalities of violence against women in Ecuador: a cross-sectional study. International Journal for Equity in Health. 2021;20(1):1-11. https://doi.org/10.1186/s12939-021-01456-9

38. Memiah P, Ah Mu T, Prevot K, Cook CK, Mwangi MM, Mwangi EW, et al. The Prevalence of Intimate Partner Violence, Associated Risk Factors, and Other Moderating Effects: Findings From the Kenya National Health Demographic Survey. Journal of Interpersonal Violence. 2021;36(11-12):5297-317. doi:10.1177/0886260518804177

39. Koenig MA, Ahmed S, Hossain MB, Khorshed Alam Mozumder AB. Women's status and domestic violence in rural Bangladesh: individual- and community-level effects. Demography. 2003 May;40(2):269-88. doi:10.1353/dem.2003.0014

40. Semahegn A, Mengistie B. Domestic violence against women and associated factors in Ethiopia; Systematic review. Reproductive Health. 2015;12(1). https://doi.org/10.1186/s12978-015-0072-1

41. Wong SPY, Chang JC. Altered Eating Behaviors in Female Victims of Intimate Partner Violence. Journal of interpersonal violence. 2016 Dec;31(20):3490-505. doi:10.1177/0886260515585535

42. García-Moreno C, Hegarty K, D'Oliveira AFL, Koziol-Mclain J, Colombini M, Feder G. The health-systems response to violence against women. The Lancet [Internet]. 2015;385(9977):1567-79. Available from: https://www.ncbi.nlm.nih.gov/pubmed/25467583

43. Signorelli MC, Hillel S, de Oliveira DC, Ayala Quintanilla BP, Hegarty K, Taft A. Voices from low-income and middle-income countries: A systematic review protocol of primary healthcare interventions within public health systems addressing intimate partner violence against women. BMJ Open [Internet]. 2018 Mar 25;8(3):e019266. Available from: https://www.ncbi.nlm.nih.gov/pubmed/29581201

44. Ansara DL, Hindin MJ. Formal and informal help-seeking associated with women's and men's experiences of intimate partner violence in Canada. Social science \& medicine (1982). 2010 Apr;70(7):1011-8. doi:10.1016/j.socscimed.2009.12.009

45. Taft AJ, Powell RL, Watson LF. The impact of violence against women on reproductive health and child mortality in Timor-Leste. Australian and New Zealand journal of public health. 2015 Apr;39(2):177-81. doi:10.1111/1753-6405.12339

46. Shaheen A, Ashkar S, Alkaiyat A, Bacchus L, Colombini M, Feder G, et al. Barriers to women's disclosure of domestic violence in health services in Palestine: qualitative interview-based study. BMC Public Health. 2020;20(1):1-10. https://doi.org/10.1186/s12889-020-09907-8

47. Heron RL, Eisma MC. Barriers and facilitators of disclosing domestic violence to the healthcare service: A systematic review of qualitative research. Health and Social Care in the Community. 2021;29(3):612- 
30. doi:10.1111/hsc.13282

48. Atteraya MS, Gnawali S, Song IH. Factors Associated With Intimate Partner Violence Against Married Women in Nepal. Journal of Interpersonal Violence. 2015;30(7):1226-46. doi:10.1177/0886260514539845 